\title{
Intermedialität von Frames in einer Polit-Talkshow
}

\author{
Werner Holly
}

\begin{abstract}
Ausgehend von der Beobachtung, dass Frames keineswegs auf Sprachliches beschränkt werden können, wird die generelle Frage der Intermedialität von Frames behandelt, und zwar am Beispiel einer Polit-Talkshow mit zusätzlichem visuellen Einblendmaterial auf einer Projektionswand. Das Beispielmaterial, das aus der teilnehmenden Beobachtung in der Bildregie stammt, stützt nicht nur die These vom ,code'- und ,mode'-übergreifenden Charakter von Frames, es wirft auch weitere Fragen auf: Welche Bilder werden überhaupt verwendet? Wie werden entsprechende Bezugnahmen hergestellt und wie lassen sie sich beschreiben? Was sind die kommunikativen und inszenatorischen Potenziale und Risiken solcher intermedialen Frame-Aktivierungen? Es zeigt sich: Während das verbale „Framing“ weitgehend in der Kontrolle der Protagonisten bleibt, sind die kamera-inszenierten Frame-Invokationen und -Evokationen von ihnen kaum überschaubar und nicht kontrollierbar.
\end{abstract}

\section{Einleitung}

Im Jahre 1658 erscheint ein sehr einflussreiches Buch, das dazu gedacht war, Schulkinder an Wissensbestände heranzuführen, der „Orbis sensualium pictus“ des calvinistischen Theologen und Lehrers Johannes Comenius aus Böhmen: Es präsentiert Listen von (lateinischen und deutschen) Wörtern, die zu kleinen Texten gefügt sind, zusammen mit bildlichen Darstellungen in Form von Holzschnitten (s. Abb. 1). Man sieht und liest z. B. unter der Rubrik XLI „Euserliche und innere Sinnen“ einen bildlichen und zwei sprachliche Frames von Modalitäten: schematische Bilder nicht nur von Auge, Ohr, Nase, Zunge und Hand, sondern auch vom Hirn, das - wie die Textseite auf lateinisch und deutsch erklärt - für 
„die gemeine Empfindnis“, „die Einbildungskraft“ und „die Gedächtnis“ steht. Von der Einbildungskraft heißt es da, dass sie dieselben Sachen“, nämlich „die von den äusserlichen Sinnen eingebrachte Sachen“, „entscheidet“, außerdem „denket/träumet" (Comenius 1658/1978, 87).
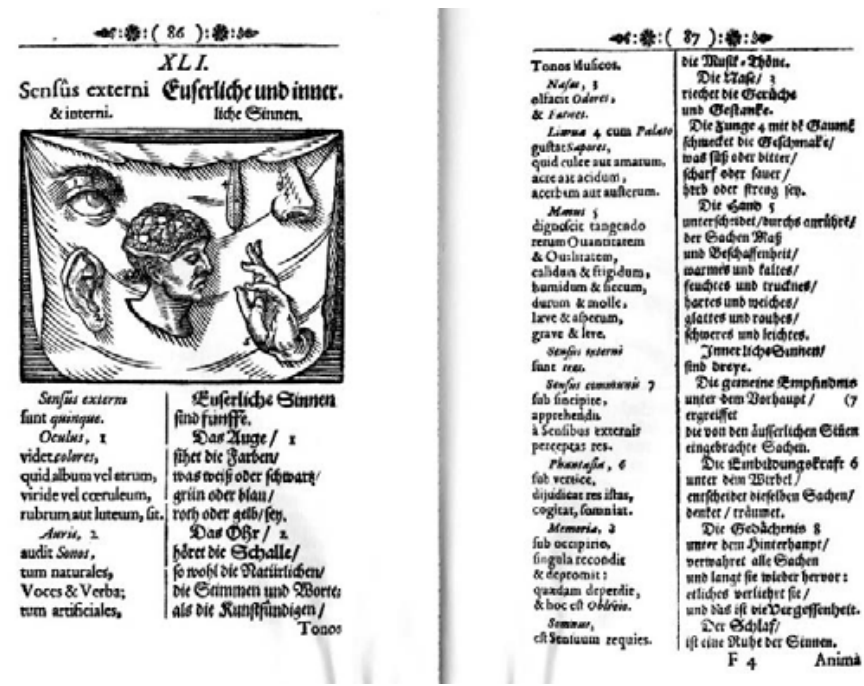

Abb. 1: Comenius, Orbis sensualium pictus, 1685 (Nachdruck 1978)

An diese Begrifflichkeiten können wir zumindest für unseren Alltagsgebrauch bis heute anschließen. Wahrnehmung und Denken (und Gedächtnis) gehören zusammen und - wie das Buch selbst vorführt - können „die Sachen“ für die Kommunikation in Wort und Bild repräsentiert oder besser konstruiert und damit kulturell verfügbar gemacht werden. So weit, so einfach. Komplizierter wird es, wenn wir weiter fragen.

Wie bauen wir Wissen auf? Manche meinen, wir beginnen mit Visuellem, mit ,Bildschemata', die sich aufgrund frühkindlicher sensomotorischer Körpererfahrungen herausbilden, also auch andere (akustische, taktile) Modalitäten einschließen (Lakoff 1987; Johnson 1987; Lakoff/Johnson 1980; dazu Konerding 1993, 59 f.; Ziem 2008, 25 ff.). Mit dem Spracherwerb treten wir dann jedenfalls ein ins Reich der Zeichen. Kaum einer würde bestreiten, dass die Strukturen unseres Wissens 
„nicht zuletzt aufgrund der Existenz und des Gebrauchs (und damit der Funktionen) sprachlicher Zeichen" entstehen - wie Busse $(2012,537)$ mit gutem Grund vorsichtig formuliert, glaubt er doch einerseits selbst, dass die Wissensstrukturen, Frames genannt, nicht einmal bei Fillmore, dem Vater der linguistischen Frametheorien, rein sprachlich bleiben können, weil „die Frame-Idee stark auf allgemeine Wissens-Hintergründe aller Art ausgedehnt wird“: „bei Fillmore verschwimmen die Grenzen zwischen ,sprachlichen' und ,kognitiven' Frames“ (ebd., 535). Andererseits scheint er eine gewisse Skepsis gegenüber Frames zu haben, die nicht an Zeichen gebunden sind: „Die Frage, welche Rollen andere Wissensquellen als die Zeichen-dominierten Quellen spielen, ob es in unserem Wissen also etwa eine ,Zeichen-freie' Ebene unmittelbar ,perzeptueller Symbole' gibt, wie es Barsalou 1993 propagiert, muss dahingestellt bleiben [...]" (ebd., 537, Anm. 2).

Die Frameforschung hat sich in ihrem linguistischen Zweig begreiflicherweise auf das Sprachliche konzentriert, ohne - wie wir gesehen haben - die Kognition ganz darauf zu beschränken. Darauf bezieht sich Meier, wenn er bemerkt, es „[...] ist zu beachten, dass das Frame-Konzept zunächst für die Ermittlung sprachlich realisierter Spuren konzeptuellen Wissens [...] entwickelt wurde, nicht jedoch für die Analyse ikonischer Zeichen“ (Meier 2010, 3).

Recht hat auch Busses Kommentar dazu, der auf die kognitivistische Tradition verweist, die auch Visuelles keineswegs ausschloss: „Der Versuch [...], das FrameModell auf die Analyse von Bildern auszuweiten, ist anregend und weist in die richtige Richtung, auch wenn seine Vermutung, dass klassische Frame-Analyse sprachzentriert sei, so keineswegs richtig ist.“" (Busse 2012, 518)

Wie es mit der Medialität oder Modalität von Frames im Detail steht, bleibt eine offene Frage. Sollen die „kognitiven“ Frames grundsätzlich an Zeichen gebunden sein und damit immer auch an Medialität, wie es bei Busse anklingt? Oder sind sie (zunächst) amodal und akodal, wie manche Kognitivisten angenommen haben (s. die Diskussion bei Schwarz 1996, 94-101)? Oder haben wir es mit einer „dualen“ Repräsentation mit (mindestens) zwei Repräsentationssystemen zu tun, wie es vor allem Paivio (z. B. 1971) vertreten hat? Es bleibt wohl vorerst ein Geheimnis, für das man auch weiter wild spekulieren kann, da man ohnehin von den Prozessen kein Bewusstsein hat, wie Schwarz (ebd., 101) schließlich folgert: 
Eine direkte Übersetzung von einem System in ein anderes setzt komplexe Übersetzungsregeln oder eine abstrakte Integratorebene voraus. Offensichtlich kann nur ein integrativer Erklärungsansatz, wonach kognitive Strukturen im LZG [Langzeitgedächtnis] sowohl modalitätsspezifisch als auch modalitätsunspezifisch repräsentiert sind, dem komplexen Phänomen gerecht werden. Wissensinhalte, die in amodalen konzeptuellen Einheiten und Strukturen gespeichert sind, sind unserem Bewußtsein jedoch nicht zugänglich. [...] Bevor konzeptuelle Wissensinhalte also unser Bewußtsein erreichen, transformiert sie ein kognitiver Prozeß in einen bestimmten Repräsentationsmodus.

Wie allerdings diese Transformation regelhaft abläuft, bleibt offen, da auch diese Übersetzungsregeln unbekannt zu sein scheinen. Hier tut sich ein regressverdächtiges Problem auf, dem man so wohl nicht entkommt. Wie solche amodalen Strukturen operieren könnten, wie dann die Bedeutungszuweisung erfolgen soll, bleibt ein Rätsel.

Die Sprachwissenschaft scheint in ihrer eigenen Domäne hier doch ein wenig weiter zu sein. Wie sprachliche Zeichen aus der Kombination kleinster bedeutungsunterscheidender Einheiten zu kleinsten bedeutungstragenden Einheiten in einem grundsätzlich dialogischen Prozess entstehen, dafür hat die Sprachwissenschaft mit Humboldt und spätestens seit der Rekonstruktion eines authentischen Saussure (Jäger 2010) ein überzeugendes Modell geliefert; das nicht-zeichenhafte Ausgangsmaterial ist aber alles andere als amodal, es ist gerade die material-modale Basis der Zeichenkonstitution.

Dass in anderen Zeichensystemen einiges anders zu sein scheint, ist ein traditionelles Thema und nicht erst (dann aber zunehmend) im Rahmen einer sich konstituierenden Bildwissenschaften klar geworden, ohne dass man vom Vorbild der Sprachwissenschaft ganz loskommt (Sachs-Hombach 2003; Kress/van Leeuwen 1996). Man muss auf jeden Fall mit autochthonen Semantiken der verschiedenen Zeichensysteme rechnen, die ja auch deren komplementäre Leistungen ausmachen. Man kann sich aber durchaus vorstellen, dass das intermediale $\mathrm{Zu}$ sammenspiel verschiedenartiger Zeichen als Verfahren der ,Transkription', wie es Ludwig Jäger in einigen Arbeiten umrissen hat (z. B. Jäger 2002; 2004), nach dem Modell der Bezugnahme von Zeichen auf Zeichen funktioniert, das ja auch innerhalb von Zeichensystemen erklärt, wie Bedeutungsgenese (über weite Strecken) ohne Referenzen auf eine zeichenexterne Welt möglich ist, also außerhalb repräsentationstheoretischer Semiotiken. 
Man darf weiteren Forschungsbedarf unterstellen. Wie Wissen und Frames, Nicht-Zeichen und Zeichen (außerhalb von Sprache) genau zusammenhängen, ist eben noch nicht klar. Mit dem Begriff der Frames ist man schnell auf spekulativem Boden. Man erreicht wieder sicheren Grund, wenn man sich an beobachtbare kognitive und kommunikative Operationen hält; dort kann man mühelos feststellen, dass wir Wissensstrukturen auf jeden Fall mit Sprache, aber eben auch in anderen Medialitäten konstruieren und bearbeiten, nicht selten in multikodaler (und multimodaler) Kombination. Dies zeigt anschaulich der „Orbis pictus“, dem viele andere Texte mit Illustrationen folgen, wie jedes bebilderte Schulbuch, jede Enzyklopädie bis hin zu Wikipedia belegt. Wir finden solche Kombinationen von sprachlichen und bildlichen Frameoperationen in vielerlei technisch-medialer Gestalt. Im Folgenden werde ich beschreiben, wie Bilder-Frames nahezu unmerklich als zusätzliches symbolisches Material in der medientechnischen Inszenierung und Kontrolle einer TV-Polit-Talkshow benutzt werden, um noch mehr Perspektivierungen und Interpretationsoptionen in den hochkomplexen multikodalen und multiauktorialen Prozess der Bedeutungsgenese einzubringen.

Dabei gehe ich von der trivialen Beobachtung aus, dass wir offensichtlich kein Problem damit haben, sprachliche Ausdrücke oder Texte mit Bildern in Verbindung zu bringen und umgekehrt, allerdings sind die Verbindungen unterschiedlichster Art. Meine These wird sein, dass Frames in der Bezugnahme über kodale und modale Grenzen hinweg scheinbar mühelos verwendet werden (Transkriptivität) und so für intermediale Kohärenzbildungen sorgen können. Die offene Frage ist, wie die Modellierung dieser Praxis aussehen könnte. Den Anfang können genauere Beschreibungen von Beispielen dieser Praxis machen. Interessant erscheint auch, ob sich Verfahren und Potenziale der intermedialen Frameverwendung feststellen lassen?

Meine ad-hoc-Analyse eines kleinen Beispiels aus einer Talkshowsendung, die eine Bildwand im Hintergrund verwendet, um zusätzliche thematische Anreize zu geben, die als Frame-Material gedeutet werden können, benutzt das FrameErschließungsverfahren von Konerding (1993), das eigentlich für die Lexikographie entwickelt worden ist. Nach meiner Überzeugung sind solche Frame-Rekonstruktionen grundsätzlich nur eine situative „Momentaufnahme“, da wir semantisches Material jeweils nach kommunikativem Bedarf immer wieder neu anord- 
nen können, was den mehr oder weniger festen Angaben, die wir von Wörterbüchern erwarten gar nicht entspricht. Es gibt deshalb auch keine Gewähr dafür, dass die dargestellten Framestrukturen mehr als plausibilisierbar werden könnten, sie bleiben notwendig Gegenstand interpretativer Auseinandersetzung, andernfalls gäbe es kaum die (kommunikativ konstitutive) Möglichkeit von Missverstehen, produktivem Streit und entsprechendem Klärungsbedarf.

Was die Reichweite solcher semantischen Spielräume angeht, muss es andererseits gewisse Grenzen geben, sonst bestünde wohl keinerlei Chance auf ein (auch nur) beschränktes Verstehen. Hier hat Konerding mit seinem Verfahren zur Gewinnung von Matrixframes, die wiederum zu bestimmten Fragen und Prädikatoren führen, ein einigermaßen handliches Modell geliefert, das es erlaubt, empirisch auffindbare semantische Bezugnahmen als Frame-Ausgestaltungen zu identifizieren. Dass bestimmte bildliche und sprachliche Elemente hier als thematisch zusammengehörig verstanden werden, belegt ihre Verwendung. Dass dies nachvollziehbar ist, belegt der Nachweis entsprechender Strukturen nach dem Konerdingschen Modell.

\section{Workplace-Studie: „Bildwandmaterial“ (Maybrit Illner 26.5.2011)}

Das empirische Material, das ich nun erläutern werde, ist am 26.5.2011 im Rahmen einer kleinen Workplace-Studie in der Bildregie des Berliner ZDF-Studios während einer Maybrit-Illner-Talkshow erhoben worden. Im Rahmen von Überlegungen zur bildlichen Inszenierung in Polit-Talkshows (s. auch Holly 2010; 2012; 2015) sollte durch die teilnehmende Beobachtung und Tondokumentation der Produktion einer Sendung folgenden Fragen nachgegangen werden:

- Wie wird das Thema inszenatorisch aufbereitet und kontrolliert?

- Mit welchen sprachlichen und bildlichen Mitteln?

- Welche Rolle spielen dabei Frames?

- Wie kann man Verfahren der intermedialen Frame-Verwendung und ihr intermediales Potenzial beschreiben?

Zum institutionellen Kontext muss man erwähnen, dass im Jahre 2011 im Vorfeld einer Neuordnung der ARD-Talkshowstruktur das konkurrierende ZDF sein 
Flaggschiff, die donnerstägliche sehr erfolgreiche Polit-Talkshow „Maybrit-Illner“, mit einem neuen Setting versah (s. Abb. 2). Dazu wurde die Sendung aus dem Innenhof des Berliner ZDF-Gebäudes in ein Studio verlegt; anstelle der sichelförmigen Sitzanordnung ohne Tische wurde ein großer runder Tisch mit einem vorderen offenen Sektor installiert. Die Zahl der Kameras wurde auf sieben erhöht: vier fahrende, eine Steadycam (5), ein Kran (6), eine von der Decke hängende (nur für den Schuss auf Maybrits Tablet, auf dem sie Einspieler startet) (7). Die größte Herausforderung für die Regie war aber eine neue Projektionswand im Hintergrund, die ständig mit zusätzlichem Bildmaterial bespielt wird: insgesamt eine gesteigerte Komplexität der Gesamtinszenierung.



Abb. 2: Setting der Maybrit-Illner-Sendung vom 26.5.2011 (mit Bildwand 1)

Das Thema der beobachteten Sendung lieferte der damals aktuell noch laufende und medienwirksame Prozess des TV-(Wetter-)Moderators Jörg Kachelmann, der wegen Vergewaltigung angeklagt und dann später vom Vorwurf freigesprochen wurde; der Titel der Sendung war: „Der Fall Kachelmann - schon jetzt ein Justizskandal?“. Als Gäste waren in der Sendung eingeladen (s. Abb. 2, von links, Illner als 3. v. 1.):

- Rüdiger Bagger (ehemaliger Staatsanwalt)

- Alice Schwarzer (,Emma'-Herausgeberin, die für die ,Bild'-Zeitung vom Prozess berichtete)

- Gerhard Strate (Staranwalt)

- Hans-Hermann Tiedje (ehemaliger ,Bild'-Chefredakteur)

- Mark Benecke (Kriminalbiologe) 
In der ersten Publikumsreihe und zwischendurch an einem Stehpult interviewt (ohne Abb.):

- Sabine Hartwig (Vertreterin der Opfer-Organisation „Weißer Ring“)

Angesichts der gesteigerten Komplexität des Produktionsablaufs erscheint ein gesteigerter Aufwand an Inszenierungskontrolle erforderlich, um dem Zuschauer eine reibungslose Präsentation einer spannenden und inhaltsreichen, zugleich aber doch gut strukturierten Aufbereitung des Themas zu ermöglichen.

Im Zentrum der Sendung steht die Moderatorin, die zugleich führungsstark und charmant Teilthemen prozessiert. Damit dies möglichst effektvoll gelingt, wird eine Reihe von Vorkehrungen getroffen, allerdings in einer sehr diskreten Form, die den Eindruck einer lebendigen und spontanen Diskussion nicht allzu sehr beeinträchtigt. Die Elemente dieser Inszenierungskontrolle sind vielfältig. Dazu gehören Ablaufpläne in Form schriftlicher Skripts mit möglichen Fragen in einer bestimmten Reihenfolge. Vor der Sendung findet ein Probedurchlauf statt, wobei Studierende als Vertreter der Gäste fungieren und erstaunlich sachkundig agieren. Zum Konzept gehört auch eine sogenannte „offene Regie“, d. h. die wichtigen professionellen Protagonisten der Produktion (Moderatorin, Regie, Kameraleute, Aufnahmeleiterin im Studio) sind mit In-Ear-Monitoring miteinander verbunden und können auf einer zweiten Kommunikationsebene ohne Beobachtung des Publikums online den Inszenierungsfortgang steuern. Das ist besonders wichtig für das ständige Antizipieren möglicher Fortsetzungen, das für den reibungslosen Einsatz von Kameras, Umschnitt, Inserts (mit Namen, Funktionen der Gäste und thematischen Kommentaren), Einspielern und das Aufspielen von Bildwandmaterial unumgänglich ist und vor allem für rasche, flexible Korrekturen bei unvorhergesehene Verläufen.

Ein starkes Instrument für die Ablaufkontrolle ist also der Einsatz der sogenannten Einspieler, also kurzer Filme, die Themenaspekte aus Sicht der Redaktion perspektivieren und die nicht selten dazu genutzt werden, eine Diskussion, die ein bisschen „aus dem Ruder läuft“, wieder in vorgesehene Bahnen zurückzulenken. Dagegen ist das Bildwandmaterial eher geeignet, als „Hintergrund“ im wörtlichen Sinne, bestimmte Aspekte eines Themas zu verstärken und mit bestimmten Assoziationen zu kontextualisieren. Wie das in unserer Beispielsendung vonstatten geht, soll im Weiteren analysiert werden. 


\subsection{Welche Bilder werden verwendet?}

Insgesamt werden sechs der vorbereiteten Stand-Bilder eingesetzt, die verschiedene Subthemen des Kachelmann-Prozesses betreffen und die ich hier als BilderFrames behandeln will. Sie sind auf den folgenden Abbildungen zu sehen, so wie sie auf der Bildwand hinter dem Tisch mit den Teilnehmern erscheinen. Das erste davon ist gewissermaßen das Standardbild (s. o., Abb. 2): Es zeigt eine Fotocollage mit Titelseiten verschiedener Presseerzeugnisse und wird in der internen Kommunikation vom Regisseur auch entsprechend genannt: die zeitungen, die fotostrecke, diese schlagzeilen-collage (Bildwand 1).

Drei weitere Bilder zeigen den Haupt-Protagonisten des Themas, Jörg Kachelmann, in verschiedenen Situationen; sie werden vom Regisseur folgendermaßen identifiziert (Abb. 3-5): son Kachelmann-bild, Kachelmann mit verteidigung (Bildwand 2); des andere Kachelmann-bild, nur kachelmann (Bildwand 3); Kachelmann abgeführt (Bildwand 4):

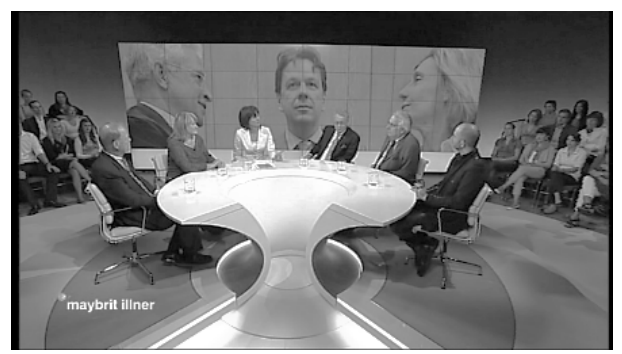

Abb. 3: Bildwand 2

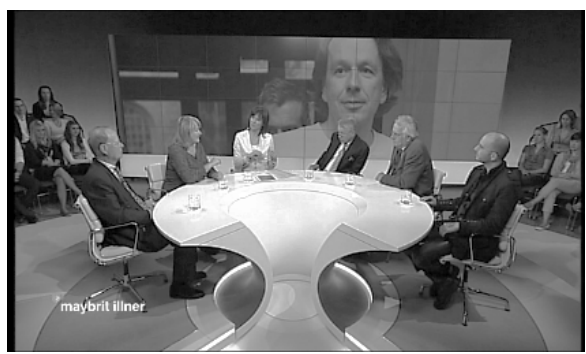

Abb. 4: Bildwand 3

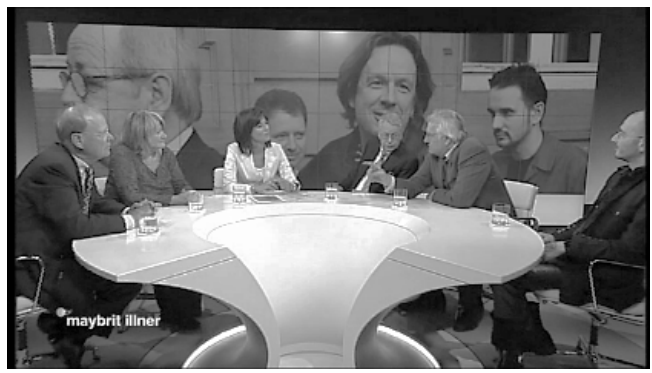

Abb. 5: Bildwand 4 
Weitere zwei Bilder sind unterschiedlicher Natur (Abb. 6-7). Das eine zeigt die Justiz allegorisch, vom Regisseur kurz iustitia genannt (Bildwand 5), das andere die Hauptfigur eines mehr oder weniger ähnlichen Falles, den französischen Politiker Dominique Strauss-Kahn, intern als DSK vor gericht bezeichnet (Bildwand 6).

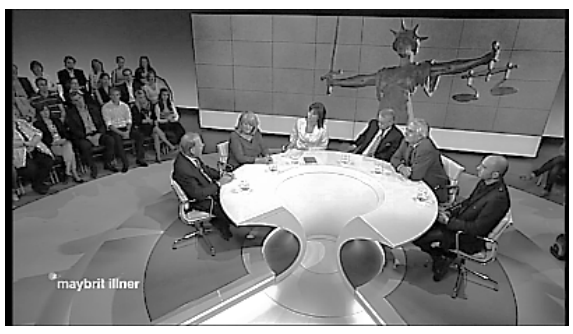

Abb. 6: Bildwand 5

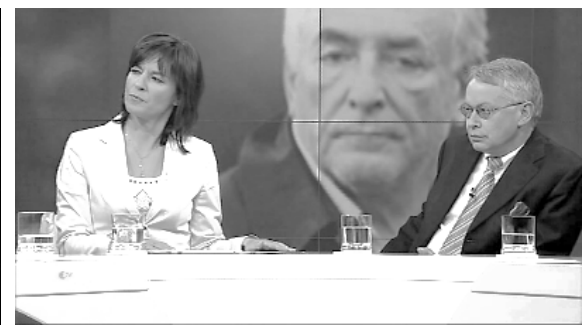

Abb. 7: Bildwand 6

Stellt man sich zunächst die Frage, wie es dazu kam, dass gerade diese Bilder vorbereitet wurden, kann man auf eine Unterscheidung Fillmores zurückgreifen, die auch Ziem heranzieht und die in folgenden beiden Zitaten erläutert wird:

Interpretative frames can be introduced into the process of understanding a text through being invoked by the interpreter or through being evoked by the text. A frame is invoked when the interpreter, in trying to make sense of a text segment, is able to assign it an interpretation by situating its content in a pattern that is known independently of the text. A frame is evoked by the text if some linguistic form or pattern is conventionally associated with the frame in question. (Fillmore 1985, 232; zit. n. Ziem 2008, 232)

Invoked frames can come from general knowledge, knowledge that exists independently of the text at hand, or from the ongoing text itself. (Fillmore, ebd.; zit. n. Ziem, ebd., 235)

Obwohl man der Ansicht sein kann, dass diese Unterscheidung von „invoked/abgerufenem“ Frame vs. „evoked/aufgerufenem“ Frame in vielen Fällen nicht sehr trennscharf gehandhabt werden kann (dazu auch Busse 2012, $203 \mathrm{ff}$.), mag sie in unserem Fall hilfreich sein, da es bei der Bereitstellung der Bilder zweifelsfrei nicht um Frames aufgrund einer konkreten Textvorlage gehen kann, sondern um Frames, die auf der Grundlage des bisherigen Diskurses zum Kachelmann-Fall interpretativ konstruiert werden, es sind also „invoked/abgerufene“ Frames, die als Belege dafür dienen können, dass Diskurse nicht allein durch sprachliche Kon- 
struktionen geprägt werden, sondern immer schon auch durch bildliche Darstellungen unterschiedlicher Abstraktionsgrade. ,Invoked“ Frames sind übrigens in der gesamten Inszenierungsvorbereitung im Spiel, so z. B. auch wenn Personen eingeladen, Subthemen für Fragen ausgewählt, Einspieler vorbereitet oder sogenannte „Bauchbinden“, also Inserts mit Namen, Funktionen usw., formuliert werden.

Im Zuge der verstärkten Beachtung piktorialer Kommunikation hat man sich in den letzten Jahren mit dem Einfluss der Bilder in verschiedenen Wissenschaften beschäftigt, auch in der Politikwissenschaft (z. B. Hofmann 1999), der Geschichte (z. B. Burke 2003; Brandt 2010; Paul 2006) oder der Soziologie (z. B. Raab 2008; Lucht/Schmidt/Tuma 2013), in den neueren Medienwissenschaften ohnehin, dort auch mit explizitem Bezug zu Frames unter dem Stichwort „Visual Framing“ (z. B. Geise/Lobinger 2013), wobei man sich mit dem ,Framing'-Begriff meist auf Goffman bezieht.

Versucht man nun in einer weiteren sprachlichen Transkription dieser Bilder (nach der, die vom Regisseur beim Abruf getätigt wird), einen gemeinsamen Frame zu konstruieren, könnte man in einem an Konerding (1993) angelehnten Verfahren für diese 6 Bilder folgende Struktur annehmen (s. Abb. 8):

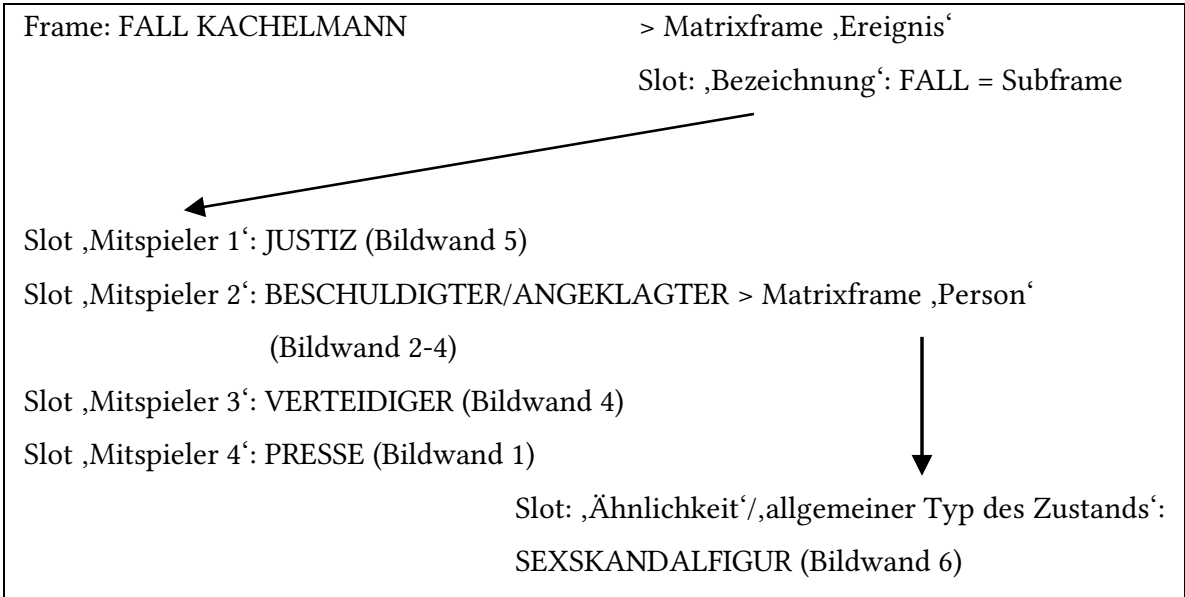


Der Gesamt-Frame (token) ist der FALL KACHELMANN (Matrixframe ,Ereignis ') mit einem Slot ,Bezeichnung' und dem Filler FALL (type), der zugleich als Subframe dient; zu diesem Subframe gehören vier Slots ,Mitspieler': der erste dieser ,Mitspieler'-Slots hat den Filler JUSTIZ (Bildwand 5), der zweite den Filler BESCHULDIGTER/ANGEKLAGTER (mit dem Matrixframe ,Person') (Bildwand 2-4), der dritte den Filler VERTEIDIGER (sichtbar auf Bildwand 2), der vierte den Filler PRESSE (Bildwand 1). Über den Matrixframe ,Person' kommt man zu einem Subframe ,Person in besonderem Zustand' mit einem Slot ,Ähnlichkeit‘, ,allgemeiner Typ des Zustands' und dem Filler SEXSKANDALFIGUR (Bildwand 6).

Man kommt - so Konerding (1993) - zu den Slots durch den Zugriff auf Matrixframes, denen man entsprechende Suchfragen zuordnen kann. So gehört zum Matrixframe ,Ereignis’ die Frage: „Welche wesentlichen Mitspieler/Interaktionspartner fungieren in dem Ereignis?“; der Matrixframe „Person’ (in besonderem Zustand) führt zur Frage: „Welchen Zuständen anderer Art ist der betreffende Zustand ähnlich [...]?" (Konerding 1993, 324, 335).

Die so gewonnene Struktur zeigt, dass die Bildauswahl zunächst einen relativ simplen Frame verwendet, der im Wesentlichen auf Mitspieler eines Justizfalles zurückgreift, das ist einmal die Justiz selbst als Institution, das sind wesentliche Rollen wie Angeklagter und Verteidiger, und das ist die Presse als Öffentlichkeitsfaktor. Nur das letzte Bild mit Strauss-Kahn benutzt eine speziellere Framebeziehung, die auf eine Ähnlichkeit der Person des Angeklagten (und der Beschuldigung) referiert, die Eigenschaft, eine prominente Figur in einem Sexskandal zu sein. Zugleich wird deutlich, dass - wie schon sprachlich - auch bildlich der Namensgeber des Falles, der Angeklagte, im Zentrum der Framekonstruktion steht, indem er nicht nur übergroß auf drei Bildern in seiner Prozessrolle zu sehen ist, sondern auch noch mehrfach auf dem Standardbild, das eigentlich Presse thematisieren soll.

\subsection{Wie werden die Bilder ab-/aufgerufen?}

Wie die Bilder im Einzelnen ab- bzw. aufgerufen werden, lässt sich anhand der Tonaufnahmen im Regieraum gut rekonstruieren. Der Regisseur folgt aufmerksam den sprachlichen Äußerungen der Talkshowteilnehmer, dabei wirken bestimmte Ausdrücke als Frameauslöser, die eines der Bilder aus dem vorbereiteten Bilderframe aktivieren. Hier handelt es sich nun z. T. um ,evoked'/aufgerufene 
Frames, z. T. um ,invoked“/abgerufene Frames bzw. Subframes. Hierzu einige Beispiele.

In einem längeren Statement zur grundsätzlich schwierigen Beweislage in Vergewaltigungsprozessen erwähnt Alice Schwarzer den Namen des französischen Politikers Dominique Strauss-Kahn, der nach Bekanntwerden von Vergewaltigungsvorwürfen - es ging um ein Zimmermädchen eines New Yorker Hotels seine geplante Präsidentschaftskandidatur aufgeben musste (// Umschnitt; \# zeitgleicher Einsatz der Regisseuräußerung):

\begin{tabular}{|c|c|}
\hline Alice Schwarzer & $\begin{array}{l}\text { Regisseur/Bild- } \\
\text { wand }\end{array}$ \\
\hline $\begin{array}{l}\text { [...] äh äh wir kennen die hohen zahlen von vergewaltigungen } \\
\text { und wir wissen // heute das zeigen die nationalen studien die } \\
\text { internationalen jeder zweite vergewaltigung passiert innerhalb } \\
\text { einer // beziehung also durch den eigenen ehemann oder freund } \\
\text { und wie sie eben schon sachten wird dann natürlich // die be- } \\
\text { weislage ganz schwierig herrn strauss-kahn wird es schwerfal- } \\
\text { len \# äh überzeugend darzulegen dass ihn äh die schwarze putz- } \\
\text { frau innerhalb einer halben stunde überfallen hat und sich // } \\
\text { sein glied inn mund gesteckt hat und dann extra schreiend raus- } \\
\text { gelaufen ist das wird schwierig aber wenn // eine beziehung is } \\
\text { kann \# natürlich dann kamma auch sagen // dass dann sacht der } \\
\text { eine das war doch einvernehmlicher sex und die andere sacht } \\
\text { ich bin vergewaltigt worden // das macht diesen fall so schwierig }\end{array}$ & $\begin{array}{l}\text { \# strauss-kahn } \\
\text { dsk vor gericht } \\
\text { wechsel jetzt? ja } \\
\text { wechsel - okay } \\
\text { achtung für die } \\
\text { drei und die drei \# } \\
\text { da isser ja }\end{array}$ \\
\hline
\end{tabular}

Es gibt unterschiedliche Evokations- und Invokationsmechanismen. Hier wird das Bild in einem simplen Verfahren gewissermaßen direkt „vom Text“ aufgerufen. Kaum fällt der Name, reagiert der Regisseur. Der Name identifiziert die Person gewissermaßen genau wie das Bild. Der Einsatz auf der Bildwand erfolgt dann in mehreren Zügen. Nachdem das Bild mit einer Art Bezeichnung selektiert ist („DSK vor gericht“), wird die Möglichkeit des Wechsels auf der Bildwand mit Frageintonation vorbereitet (,wechsel jetzt?") und bestätigt (,ja wechsel“), dann wird die Kamera 3, welche die Bildwand erfasst, vorbereitet (,achtung für die drei“) und es wird der Umschnitt ausgelöst (,und die drei“). Man sieht nun das entsprechende Bild (Abb. 7), wie es über den Sender geht, was der Regisseur erfreut zur Kenntnis nimmt, denn das Manöver hat geklappt: „da isser ja“.

Im nächsten Beispiel wird die Bildauswahl ein wenig schwieriger, da es um ein Kachelmann-Bild geht und unter mehreren zu wählen ist: 


\begin{tabular}{|c|c|}
\hline Maybrit IIlner / Alice Schwarzer & Regisseur/Bildwand \\
\hline $\begin{array}{l}\text { I: Alice Schwarzer wir ham sie zitiert mit dem } \\
\text { satz dass wenn es einen freispruch nur aus } \\
\text { mangel an beweisen gäbe dann wäre das eine } \\
\text { katastrophe in ihren augen warum eigentlich } \\
\text { gilt nicht ne unschuldsvermutung für jeman- } \\
\text { den } \\
\text { S: woher ham sie den } \\
\text { I: der hat gestanden in der Emma und wenn } \\
\text { sie jetzt wollen } \\
\text { S: is das wahr ja das könn wer vorlesen (I: ja) } \\
\text { daran sieht man zum beispiel an diesem satz } \\
\text { äh äh wie dreist der Kachelmann-verteidi- } \\
\text { ger ständig zu lügen pflegt er hat diesen satz } \\
\text { zitiert \# in seinem plädoyer wo ich auf der } \\
\text { pressebank saß da konnt ich sagen hallo alles } \\
\text { falsch und hat [...] }\end{array}$ & $\begin{array}{l}\text { \# äh ich brauch mal so'n Kachel- } \\
\text { mann-bild mit ähm Kachelmann } \\
\text { mit verteidigung }\end{array}$ \\
\hline
\end{tabular}

Das hier verwendete Bild (Abb. 3) könnte man als Ikon des Kompositums deuten, denn es zeigt Kachelmann zwischen seinen Verteidigern, bildet also gewissermaßen die zwei Komponenten des Kompositums ab; umgekehrt hat offensichtlich die Verwendung dieses Kompositums unmittelbar dieses Bild aufgerufen. Der Regisseur nähert sich der Auswahl in zwei Stufen, zunächst wird die Gruppe der entsprechenden Bilder ausgewählt („so’n Kachelmann-bild“), dann die genauere ,Bezeichnung’ („Kachelmann mit verteidigung“).

Im nächsten Fall ist die Verknüpfung von Sprechtext und Bildauswahl viel abstrakter. Der ehemalige Staatsanwalt Bagger äußert in Anbetracht der Beweislage Verständnis für die Schwierigkeiten eines Richters (,ich möchte kein richter sein“), was beim Regisseur dazu führt, die Thematisierung eines generellen Justizproblems durch die Auswahl einer allegorischen Darstellung der „Iustitia“ (Abb. 6) zu unterstützen und verstärken:

\begin{tabular}{|l|l|}
\hline Rüdiger Bagger / Maybrit Illner & Regisseur/Bildwand \\
\hline B: [...] dies is ein fall sui generis aus zwei din- \\
gen wir ham in einem stillen zimmer zwei \\
leute einer beschuldigt ein opfer \\
I: einer sacht ja einer sacht nein // keine zeu- \\
gen & \\
\hline
\end{tabular}


B: genau aussage gegen aussage keine zeugen der satz war gut ich möchte kein richter sein der is gut die ham ein ganz ganz \# schwere aufgabe und die wird ihnn durch die medien zum teil noch erschwert weil zeuginnen die dann aussagen sollten vorher schon in dem medien gegen viel geld ihre ganzen \# intimen sachen ausgebreitet ham ...
\# packen wir iustitia rauf bitte schnell warte warte - jetzt

\# KAMERA ZEIGT iustitia AUF BILDWAND

Hier kann man davon sprechen, dass das Bild nicht unmittelbar ausgelöst wird, sondern dass mehr interpretative Leistung des Regisseurs nötig ist, um den Subframe ,Justiz' abzurufen. Im nächsten Beispiel ist dieser Interpretationsaufwand noch größer. Man fragt sich, was hier den Regisseur dazu bewegt, ein neutraleres Kachelmann-Bild (Abb. 4) abzurufen („xxx“ für in der Aufzeichnung unverständliche Passage):

\begin{tabular}{|l|l|}
\hline Alice Schwarzer /Gerhard Strate & Regisseur/Bildwand \\
\hline S: [..] äh ausschließlich der von dem gericht zu & \\
recht abgelehnte voreingenommene gutachter & \\
Brinkmann ich kenne teile des gutachtens in & \\
der tat erstaunlich dass ein so seriöser mann & \\
ein so voreingenommenes // gutachten macht & \\
St: woher kenn sie das & \\
S: ja w w da müssen se alle kollegen immer & \\
fragen woher se was kennen von der staatsan- & \\
waltschaft nicht so ähm also alle haben im- & \\
mer gesacht kann sein \# kann nicht sein ja & \\
St: professor Püschel// sagt es sei wahrschein- & \# gib mir noch mal des andere Ka- \\
lich & chelmann des elf bild des ds nur Ka- \\
S: das problem wahrscheinlich ja aber profes- & chelmann (xxxx) glaub ich mach mal \\
sor Püschel hat auch ne große phantasie [...] & kannst jetzt wech- genau das \\
& \\
\hline
\end{tabular}

Alice Schwarzer unterstellt einem Gutachter Voreingenommenheit, was sie mit einer Aussage belegen will, die das umstrittene Gutachten mit der Ambivalenz anderer Gutachter (,alle haben immer gesacht kann sein kann nicht sein“) kontrastiert. Wenn dies nun mit einem Kachelmann-Bild kontextualisiert wird, kann man nur vermuten, dass hier die schwierige Suche der Gutachter nach der Wahrheit anhand eines mehrdeutigen Gesichtsausdrucks verdeutlicht werden soll, 
nach dem Motto: Diese Person gibt Rätsel auf. Ebenso vage wie diese Bezugnahme ist die Referenz des Regisseurs auf das Bild, in drei Schritten: (1) des andere Kachelmann, (2) des elf bild, (3) des ds nur Kachelmann (xxxxx) glaub ich. Während (1) und (2) Kontrast zum bisherigen Bildmaterial und eine interne Nummerierung bemühen, wird im Schritt (3) mithilfe einer spezifischen Differenz gearbeitet, nämlich dass es nur Kachelmann zeige (wie man die unverständliche Lücke wohl schließen muss), wobei abschließend die Vagheit mit einer Unsicherheits-Hecke („glaub ich“) noch explizit markiert wird.

Im letzten Beispiel, das ich noch anführen möchte, beruht der interpretative Abruf auf einer klassischen Ekphrasis-Episode im Statement des Anwalts Gerhard Strate, das sich mit dem höchst problematischen Sachverhalt beschäftigt, dass von den Prozessbeteiligten die „Macht der Bilder“ dazu genutzt werden kann, über die Medien auf die Stimmung in der Öffentlichkeit und damit letztlich auch auf das Gericht Einfluss zu nehmen. Strate unterstellt der Staatsanwaltschaft, dass sie durch die Bekanntgabe eines Haftprüfungstermins solche Bildanlässe inszeniert habe, wobei offen bleibt, ob dies nicht auch von der Verteidigung instrumentalisiert werden konnte:

\begin{tabular}{|l|l|}
\hline Gerhard Strate & Regisseur/Bildwand \\
\hline St: ich weiß nich also hier grade bei dieser ge- & \\
schichte iss ja so dass die staatsanwaltschaft & \\
ne pressemitteilung rausgegeben hat wo se & \\
exakt die uhrzeit und auch den ort der haft- \\
prüfung beziehungsweise der anhörung von & \\
herrn Kachelmann beschrieben hat das heißt & \\
alle wussten dort müssen wer uns versammeln & \\
und das war natürlich ein ort wo auch offen- & \\
bar keine blinde zuführung erfolgt sondern & \\
man muss eben zugeführt werden über einn & \\
derartigen mannschaftswagen äh das is si- & \\
cherlich auch \# mitzuverantworten durch die & \\
staatsanwaltschaft ich will allerdings auch & \\
nicht ausschließen dass der damalige verteidi- & \# zeigst du mir noch mal Kachel- \\
ger möglicherweise auch durchaus n gefallen & mann abgeführt wart ich geb dir $\mathrm{n}$ \\
daran gehabt hat dass das so gelaufen is aber & [...] \\
\hline
\end{tabular}

Hier liefert das Bild (Abb. 5) nicht nur die Illustration der geschilderten Szene, sodass eine Bildbeschreibung im wörtlichen Sinne daraus wird, sondern zugleich 
den Beleg für die These von der Wirkmächtigkeit von „Bildpolitik“ in spektakulären Prozessen. Mit dem Konkretum „Mannschaftswagen“ wird ein Frame aufgerufen, der sofort die Aufmerksamkeit auf ein Bild lenkt, das intern „Kachelmann abgeführt" heißt. Dabei muss das Bild, das inzwischen überall bekannt war, den Auslöser ,Mannschaftswagen’ selbst gar nicht mehr zeigen, weil man aus den Fernsehbildern des gesamten Diskurses weiß, dass dieser links vom gezeigten Bildausschnitt stand (s. Abb. 9). Im Übrigen war in der Sendung die vollständige Szene auch noch einmal in einem Einspielfilm zu sehen.

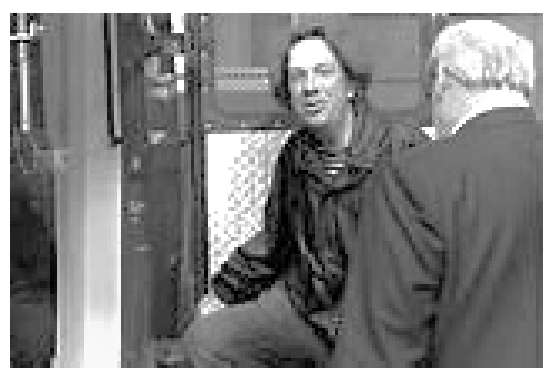

Abb. 9: Der Mannschaftswagen

Insgesamt sieht man, dass die Auslöser im Sprechtext unterschiedlicher Natur sind; sie reichen von einfachen Verbindungen zum Bild durch Namen (StraussKahn) oder Komposita (Kachelmann-verteidiger) bis zu komplizierteren Bezugnahmen, die sich auf ganze Situationen und ihre Implikationen erstrecken. Für den Rezipienten stehen Frameaufruf und Frameabruf nicht nur im Dienste des Textverstehens, sondern sie leisten einen Beitrag zur multiauktorialen und multimedialen Semiose mit reichhaltigem Interpretationspotenzial, das für die Protagonisten wiederum ein Kontrollproblem darstellt.

\subsection{Transkriptive Potenziale von Bildern?}

Allgemein gilt: Die Kameraführung in Polit-Talkshows generiert durch die Selektion von Einstellungen und Umschnitten Bedeutungskomponenten, die sprachliche Äußerungen im Sinne von Jäger (2002) „transkribieren“, d. h. überformen, implizit kommentieren und dadurch ,anders lesbar" machen. Brisant ist vor allem, dass so die alleinige Auktorialität dem Sprecher entzogen wird und er die 
„performative Letztfassung“ nicht mehr kontrolliert, sondern sich partiell an Instanzen technischer Medialität ausliefert, die auch nahezu unmerklich auf den Rezipienten wirken. Bildwandmaterial ist hierfür ein prominentes Beispiel, indem es seinerseits nicht nur stereotype Frames aufruft, sondern auch zusätzliche Bedeutungskomponenten und Perspektivierungen einbringt. Bildwandmaterial wird nach abgerufenen Frames vorbereitet, wird nach aufgerufenen und abgerufenen Frames eingeblendet und führt selbst zum Aufruf und Abruf von Frames, d. h. es steht nicht für isolierte Referenzobjekte, sondern präsentiert Frames, die sich auf andere (sprachliche und bildliche) Frames beziehen.

Man kann also auch die Auswahl und den Auf-/Abruf der Bilder als Bezugnahmepraktiken verstehen. Der Sprechertext ,transkribiert' zunächst durch Bezüge auf Frameelemente die vorbereiteten Bilder und löst deren kommunikativen Einsatz aus. In einem zweiten Schritt wirken nun die Bilder ,transkriptiv' auf den Sprechertext zurück, sodass sich eine Wechselseitigkeit der intermedialen Bezüge ergibt. Auch dies führt - wie schon beim „Orbis pictus“ - zu Framevariationen und -erweiterungen, jeweils über die modalen und kodalen Grenzen hinweg.

Im Folgenden soll noch einmal an einem Beispiel illustriert werden, wie man das transkriptive Potenzial der Bildwand-Elemente im Rahmen einer Betrachtung von Frames erfassen kann.

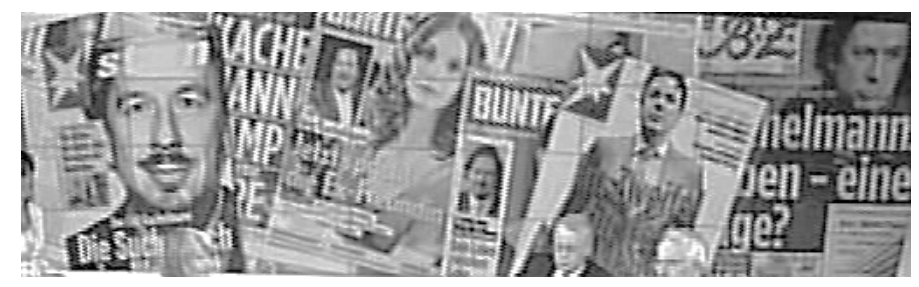

Abb. 10: „Fotostrecke“

Fragt man danach, welche impliziten/abgerufenen oder expliziten Frames durch das Standardbild an der Bildwand, die sogenannte „Fotostrecke“ (s. Abb. 10), ins Spiel kommen, so kann man interpretativ erschließen:

(1) Es wird nahegelegt, dass bebilderte Presseerzeugnisse (Illustrierte) die massenmedialen Mitspieler im Fall Kachelmann sind, andere Medien werden also ausgeblendet. 
(2) Kachelmann erscheint sichtbar als eine Figur der weniger seriösen Presse, als ein Gegenstand von Yellow-Press-Medien; somit wird er in ein Zwielicht gerückt, das seine Rolle als Angeklagter in einem Vergewaltigungsprozess zu seinen Ungunsten grundiert.

(3) Ganz wörtlich wie im übertragenen Sinne thematisiert die Fotomontage Kachelmann als einen „Mann mit vielen Gesichtern“.

(4) Nicht alle dieser Gesichter Kachelmanns sind sympathisch, man betrachte z. B. auf der linken Seite die schwarz-weiß-Frontal-Ansicht mit Bart.

Diese wenigen Hinweise mögen verdeutlichen, dass hier ein optisch eindrucksvolles Instrument vorliegt, das die Gespräche mit einem semantischen Hintergrund ausstattet; auch wenn der einzelne Zuschauer mit seinen individuellen Verstehens- und Interpretationsleistungen dies mehr oder weniger bewusst und mehr oder weniger musterhaft aufnehmen kann, entsteht so doch ein transkriptives Potenzial, das durchaus manches Gesagte mit einer gewissen Tendenz kontextualisieren kann.

\section{Fazit}

Die Frage nach dem Zusammenhang von Frames und Medialität ist nach wie vor wenig geklärt. Hier stehen sich im Wesentlichen die Positionen gegenüber, die Frames (zumindest auch) in einem amodalen Bereich lokalisiert sehen, und andere, für die Framestrukturen grundsätzlich modal und kodal verankert sind, wobei ständig transkriptive Bezüge aufeinander stattfinden.

So bietet umgekehrt die Frame-Semantik Ansatzmöglichkeiten für die Beschreibung intermedialer Bedeutungsgenese, wie sie in verschiedensten Typen von Kommunikationen mit und ohne technische Medien an der Tagesordnung sind. Dies ist besonders augenfällig in technischen Medien, in denen die einzelnen Kodes und Modes gesondert und in mehr oder weniger kontrollierbarer Gestaltung prozessiert werden müssen. Der Kode- und Modewechsel lässt sich dabei als Aufruf bzw. Abruf von Frames modellieren. Belege dafür liefert die Beobachtung von Produktionsprozessen, wie sie hier anhand von Dokumentationsmaterial aus dem Regieraum in Verbindung mit der Aufzeichnung der Sendung vorgeführt wurden. 
Dabei hat sich gezeigt: Während das verbale „Framing“ weitgehend in der Kontrolle der Protagonisten bleibt, sind die kamera-inszenierten Frame-Invokationen und -Evokationen von ihnen nicht kontrollierbar. Aber auch die Inszenierer können nicht beliebiges Bildmaterial einspielen, sondern arbeiten im nur partiell vorhersehbaren Ablauf live/online jeweils reaktionsschnell und nach aktuellen Erfordernissen. Umso mehr sind sie auf allgemeine und leicht nachzuvollziehende Wissensstrukturen angewiesen, können aber zusätzliche Perspektivierungen einbringen, indem sie das transkriptive Potenzial der Bilder nutzen.

\section{Literatur}

Barsalou, Lawrence W. (1993): Flexibility, Structure, and Linguistic Vagary in Concepts: Manifestations of a Compositional System of Perceptual Symbols. In: Alan F. Collins / Susan E. Gathercole / Martin A. Conway / Peter E. Morris (eds.): Theories of Memory. Hove, UK / Hillsdale, NJ: Lawrence Erlbaum, 29-101.

Brandt, Bettina (2010): Germania und ihre Söhne: Repräsentationen von Nation, Geschlecht und Politik in der Moderne. Göttingen: Vandenhoeck \& Ruprecht. Burke, Peter (2003): Augenzeugenschaft. Bilder als historische Quellen. Berlin: Verlag Klaus Wagenbach.

Busse, Dietrich (2012): Frame-Semantik. Ein Kompendium. Einführung - Diskussion - Weiterentwicklung. Berlin / Boston: de Gruyter.

Comenius, Johann Amos (1658/1978): Orbis sensualium pictus. Dortmund: Harenberg.

Fillmore, Charles J. (1985): Frames and the semantics of understanding. In: Quaderni di Semantica 6, 222-254.

Geise, Stephanie / Katharina Lobinger (Hrsg.) (2013): Visual Framing. Perspektiven und Herausforderungen der Visuellen Kommunikationsforschung. Köln: von Halem.

Hofmann, Wilhelm (Hrsg.) (1999): Die Sichtbarkeit der Macht. Theoretische und empirische Untersuchungen zur visuellen Politik. Baden-Baden: Nomos. 
Holly, Werner (2010): Besprochene Bilder - bebildertes Sprechen. Audiovisuelle Transkriptivität in Nachrichtenfilmen und Polit-Talkshows. In: Arnulf Deppermann / Angelika Linke (Hrsg.): Sprache intermedial: Stimme und Schrift, Bild und Ton. Berlin / New York: de Gruyter, 359-382.

Holly, Werner (2012): Transkriptiv kontrollgemindert: Automatismen und Sprach-Bild-Überschreibungen in Polit-Talkshows. In: Tobias Conradi / Gisela Ecker / Norbert Otto Eke / Florian Muhle (Hrsg.): Schemata und Praktiken. München, 161-189.

Holly, Werner (2015): Bildinszenierungen in Talkshows. Medienlinguistische Anmerkungen zu einer Form von „Bild-Sprach-Transkription“. In: Heiko Girnth / Sascha Michel (Hrsg.): Multimodale Kommunikation in Polit-Talkshows. Stuttgart: ibidem, 123-144.

Jäger, Ludwig (2002): Transkriptivität. Zur medialen Logik der kulturellen Semantik. In: Ludwig Jäger / Georg Stanitzek (Hrsg.): Transkribieren. Medien / Lektüre. München: Fink, 19-41.

Jäger, Ludwig (2004): Die Verfahren der Medien: Transkribieren - Adressieren Lokalisieren“. In: Jürgen Fohrmann / Erhard Schüttpelz (Hrsg.): Die Kommunikation der Medien. Tübingen: Niemeyer, 69-79.

Jäger, Ludwig (2010): Ferdinand de Saussure zur Einführung. Hamburg: Junius.

Johnson, Mark (1987): The Body in the Mind. The Bodily Basis of Meaning, Imagination, and Reason. Chicago: University of Chicago Press.

Konerding, Klaus-Peter (1993): Frames und lexikalisches Bedeutungswissen. Untersuchungen zur linguistischen Grundlegung einer Frametheorie und zu ihrer Anwendung in der Lexikographie. Tübingen: Niemeyer.

Kress, Gunther / Theo van Leeuwen (1996): Reading images. The grammar of visual design. London / New York: Routledge.

Lakoff, George (1987): Woman, Fire, and Dangerous Things. What Categories Reveal about the Mind. Chicago: University of Chicago Press

Lakoff, George / Mark Johnson (1980): Metaphors We Live By. Chicago: University of Chicago Press.

Lucht, Petra / Lisa-Marian Schmidt / René Tuma (Hrsg.) (2013): Visuelles Wissen und Bilder des Sozialen. Aktuelle Entwicklungen in der Soziologie des Visuellen. Wiesbaden: Springer. 
Meier, Stephan (2010): Bild und Frame. Eine diskursanalytische Perspektive auf visuelle Kommunikation und deren methodische Operationalisierung. In: Anna Duszak / Juliane House / Łukasz Kumięga (eds.): Globalization, Discourse, Media: In a Critical Perspective. Warschau: Wydawnictwo Uniwersytetu Warszawskiego, 371-392.

Paivio, Allen (1971): Imagery and Verbal Processes. New York: Holt, Rinehart and Winston.

Paul, Gerhard (2006): Visual History. Göttingen: Vandenhoeck \& Ruprecht.

Raab, Jürgen (2008): Visuelle Wissenssoziologie. Theoretische Konzeption und materiale Analysen. Konstanz: UVK Verlagsgesellschaft.

Sachs-Hombach, Klaus (2003): Das Bild als kommunikatives Medium. Elemente einer allgemeinen Bildwissenschaft. Köln: von Halem.

Schwarz, Monika (1996): Einführung in die Kognitive Linguistik. 2. Auflage Tübingen / Basel: UTB.

Ziem, Alexander (2008): Frames und sprachliches Wissen. Kognitive Aspekte der semantischen Kompetenz. Berlin / New York: de Gruyter. 

